\title{
Global Stability Analysis of SEIR Model with Holling Type II Incidence Function
}

\author{
Mohammad A. Safi ${ }^{1}$ and Salisu M. Garba ${ }^{2}$ \\ ${ }^{1}$ Department of Mathematics, The Hashemite University, Zarqa 13115, Jordan \\ ${ }^{2}$ Department of Mathematics and Applied Mathematics, University of Pretoria, Pretoria 0002, South Africa
}

Correspondence should be addressed to Mohammad A. Safi, masafi@hu.edu.jo

Received 27 June 2012; Revised 9 August 2012; Accepted 12 September 2012

Academic Editor: Jacek Waniewski

Copyright (C) 2012 M. A. Safi and S. M. Garba. This is an open access article distributed under the Creative Commons Attribution License, which permits unrestricted use, distribution, and reproduction in any medium, provided the original work is properly cited.

A deterministic model for the transmission dynamics of a communicable disease is developed and rigorously analysed. The model, consisting of five mutually exclusive compartments representing the human dynamics, has a globally asymptotically stable diseasefree equilibrium (DFE) whenever a certain epidemiological threshold, known as the basic reproduction number $\left(\mathcal{R}_{0}\right)$, is less than unity; in such a case the endemic equilibrium does not exist. On the other hand, when the reproduction number is greater than unity, it is shown, using nonlinear Lyapunov function of Goh-Volterra type, in conjunction with the LaSalle's invariance principle, that the unique endemic equilibrium of the model is globally asymptotically stable under certain conditions. Furthermore, the disease is shown to be uniformly persistent whenever $\mathcal{R}_{0}>1$.

\section{Introduction}

Mathematical models have been widely used to gain insight into the spread and control of emerging and reemerging disease. The dynamics of these models is usually determined by a threshold quantity known as the basic reproduction number (denoted by $\mathcal{R}_{0}$ ), which is defined as the number of secondary cases generated by an infected individual in a completely susceptible population [1-5]. Characteristically, when $\mathcal{R}_{0}$ is less than unity, a small influx of infected individuals will not generate large outbreaks, and the disease dies out in time. On the other hand, when $\mathcal{R}_{0}$ exceeds unity, the disease will persist. A basic epidemic model supports at least two equilibria (a disease-free equilibria and endemic equilibria); $\mathcal{R}_{0}$ plays important role in the study of equilibria of a model. Several models found in the literature [2, 4-15] have been used to show that when $\mathcal{R}_{0}$ crosses the threshold, $\mathcal{R}_{0}=1$, a transcritical bifurcation takes place. That is, asymptotic local stability is transferred from the disease-free state to the new (emerging) endemic (positive) equilibria. In some cases, it can be shown that the transfer of asymptotic stability is independent of initial conditions; that is, it is global; see, for instance, $[6,8-12,16]$. Establishing global properties of a dynamical system using Lyapunov function is generally a nontrivial problem. This is owing to the fact that there are no systematic methods for constructing Lyapunov function for infectious disease models with standard incidence rate [17]. The most successful approach to the problem is the direct Lyapunov method which involves the use of quadratic function of the form $\omega\left(x_{1}, x_{2}, \ldots, x_{n}\right)=\sum_{i=1}^{n}\left(c_{i} / 2\right)\left(x_{i}-x_{i}^{*}\right)$ or by using nonlinear Lyapunov function of Goh-Volterra type of the form $\omega\left(x_{1}, x_{2}, \ldots, x_{n}\right)=\sum_{i=1}^{n}\left(c_{i} / 2\right)\left(x_{i}-x_{i}^{*}-\right.$ $\left.x_{i}^{*} \ln \left(x_{i} / x_{i}^{*}\right)\right)$. However, other methods used in establishing global properties of some epidemic models include Dulac's criterion to eliminate the existence of the periodic solution and prove the global stability by the Poincare Bendixson theorem [18] and those reported in Kamgang and Sallet [19] and Qiao et al. [20].

Let $S(t), I(t)$, and $N(t)$ denote the number of susceptible individuals, infectious individuals, and the total size of the population at time $t$, respectively. Further, let $\beta(N)$ be the average number of contacts that is sufficient to transmit infection (effective contact rate). Then, the force of infection, given by $\beta(N) I / N$, represents the average number of contacts a susceptible individual makes with infectious individuals per unit time. If $\beta(N)=\beta N$ (i.e., the contact rate depends 
on the total population, $N)$, then the incidence function $g_{1}(I)=\beta I$ is called mass action incidence. If $\beta(N)=\beta$ (a constant), then the incidence function $g_{2}(I)=\beta I / N$ is called standard incidence [4]. These two functions are widely used in modeling the transmission dynamics of the human diseases $[1,21]$. Another widely used incidence function is the Holling type II incidence function, given by $g_{3}(I)=$ $\beta I /(1+\omega I)$, with $\omega>0$, [22-28].

The nonlinear incidence function of type $g_{3}(I)$ was first introduced by Capasso and Serio [22], in their study of cholera epidemic. The main justification for using such a functional form of the incidence function stems from the fact that the number of effective contacts between infective individuals and susceptible individuals may saturate at high infective levels due to crowding of infective individuals or due to the preventive measures (and behavioral changes) taken by the susceptible individuals in response to the severity of the disease [23, 25-27].

A number of mathematical models have been developed in the literature to gain insights into the transmission dynamics of diseases with subpopulation (compartments) [4, 6-14, 29-31]. The choice of which compartment to include in a model depends on the characteristic of the particular disease being modelled and the purpose of the model [4].

The classical SIR or SIRS model assumed that the disease incubation is negligible so that each susceptible individual (in the $S$ class) once infected becomes infectious (and move to $I$ class) and later recovers (to move to $R$ class) where they acquire permanent or temporary immunity [32]. However, more general models than SIR or SIRS models assumed that susceptible individuals, once infected, first go through the latent period (in the $E$ class) before becoming infectious; the resulting models are of SEIR or SEIRS type, depending on whether recovered individuals acquired permanent or temporary immunity $[33,34]$.

The model considered in this study is based on SEIR ( $S$ : susceptible, $E$ : exposed, $I$ : infected, $R$ : recovered) where recovered individuals acquire permanent immunity, so that they will not become infected again. This is owing to the fact that for many viral diseases such as measles, smallpox, rubella, HIV/AIDS, and influenza recovered individuals confer lifelong immunity [29, 33]. Huang and Takeuchi [29] study the classical SIR, SIS, SEIR, and SEI models with time delay and a general incidence function. Safi et. al. [13] consider the effect of periodic fluctuations on the transmission dynamics of a communicable disease using SEIRS model, subject to quarantine and isolation; the authors show that adding periodicity to the autonomous model does not alter the threshold dynamics of the model with respect to the control of the disease in the population. Zhang and $\mathrm{Ma}$ [35] considered the global dynamics of an SEIR model with saturating contact rate. Korobeinikov [36] established global asymptotic dynamics of SEIR and SIR models with several parallel infectious stages. Li et al. [33] analyzed the global dynamics of an SEIR model with vertical transmission and bilinear incidence. Li and Jin [34] consider global stability of an SEIR epidemic model with infectious force in latent, infected, and immune period. It should be stated, however, that the aforementioned three studies $[33,34,36]$ considered mass action (bilinear) incidence to model the infection. This study focuses on the mathematical modeling of the transmission dynamics of an arbitrary disease with educated (counsel) and uneducated infectious stages.

This study has three important differences from those reported in $[33,34,36]$. The first is established essential qualitative features of an SEIR model with Holling type II incidence function. The second is that a public health education and counselling are offered to infected individuals $\left(I_{u}\right)$. Recent studies further reinforced the widely held belief that one key strategy for preventing and controlling the spread of communicable disease such as HIV (especially in resource-poor nations) is to provide HIV-related public health education and counselling (such as sexual education and awareness of the risk and life-threatening consequences of HIV/AIDS) which would, hopefully, lead to reduction in risky sexual behavior and safer lifestyle within the community [7]. In addition to the above extensions, rigorous qualitative analysis will be provided for the resulting SEIR model. In particular, the paper gives special emphasis on the global asymptotic stability of the disease-free equilibrium and endemic equilibrium.

The paper is organized as follows. The model is formulated and analysed for its basic dynamical features in Section 2. The stability analysis of the model is presented in Sections 3 and 4.

\section{Model Formulation}

The total population at time $t$, denoted by $N(t)$, is subdivided into five compartments of susceptible $(S(t))$, exposed (those who have been infected but are not yet infectious) $(E(t))$, uneducated infected individuals $\left(I_{u}(t)\right)$, educated infected individuals $\left(I_{e}(t)\right)$, and recovered $(R(t))$ individuals, so that

$$
N(t)=S(t)+E(t)+I_{u}(t)+I_{e}(t)+R(t) .
$$

The susceptible population is increased by the recruitment of individuals into the population (assumed susceptible), at a rate $\Pi$. Susceptible individuals may acquire infection, following effective contact with infected individuals (in the $I_{u}$ or $I_{e}$ class) at a rate $\lambda(t)$, where

$$
\lambda(t)=\beta\left(\frac{I_{u}}{1+\alpha_{1} I_{u}}+\frac{\eta I_{e}}{1+\alpha_{2} I_{e}}\right) .
$$

In (2), $\beta$ is the effective contact rate (contact capable of leading to infection), while the modification parameter $0<\eta<1$ accounts for the assumed reduction in disease transmission by educated infected individuals in comparison to uneducated infected individuals in the $I_{u}$ class. The population of susceptible individuals is further decreased by natural death (at a rate $\mu$ ). Thus, the rate of change of the susceptible population is given by

$$
\frac{d S}{d t}=\Pi-[\mu+\lambda(t)] S(t)
$$

The population of exposed individuals is generated by the infection of susceptible individuals (at the rate $\lambda(t)$ ). This 
population is decreased by development of disease symptoms (at a rate $\kappa$ ) and natural death (at a rate $\mu$ ), so that

$$
\frac{d E}{d t}=\lambda(t) S(t)-(\kappa+\mu) E(t)
$$

The population of uneducated infected individuals is generated at the rate $\kappa$. It is decreased by natural recovery (at a rate $\gamma_{1}$ ), education (at a rate $\sigma$ ), natural death (at the rate $\mu$ ), and disease-induced death (at a rate $\delta_{1}$ ). This gives

$$
\frac{d I_{u}}{d t}=\kappa E(t)-\left(\sigma+\gamma_{1}+\mu+\delta_{1}\right) I_{u}(t)
$$

The population of educated infected individuals is generated by the education of infected individuals (at the rate $\sigma)$. This population is decreased by recovery (at a rate $\gamma_{2}$ ), natural death (at the rate $\mu$ ), and disease-induced death (at a rate $\delta_{2}<\delta_{1}$ ). It is assumed that the disease-induced mortality rate of educated infected individuals is low in comparison with uneducated infected individuals. Hence, the rate of change of this population is given by

$$
\frac{d I_{e}}{d t}=\sigma I_{u}(t)-\left(\gamma_{2}+\mu+\delta_{2}\right) I_{e}(t) .
$$

Finally, the population of recovered individuals is generated by the recovery of uneducated and educated infected individuals (at the rates $\gamma_{1}$ and $\gamma_{2}$, resp.). It is decreased by natural death (at the rate $\mu$ ), so that

$$
\frac{d R}{d l t}=\gamma_{1} I_{u}(t)+\gamma_{2} I_{e}(t)-\mu R(t) .
$$

Thus, the model for the transmission dynamics of an infectious disease in the presence of educated (counsel) infected individuals is given by the following nonlinear system of differential equations:

$$
\begin{aligned}
& \frac{d S}{d t}=\Pi-[\mu+\lambda(t)] S(t), \\
& \frac{d E}{d t}=\lambda(t) S(t)-[\mu+\kappa] E(t), \\
& \frac{d I_{u}}{d t}=\kappa E(t)-\left[\mu+\sigma+\gamma_{1}+\delta_{1}\right] I_{u}(t), \\
& \frac{d I_{e}}{d t}=\sigma I_{u}(t)-\left[\mu+\gamma_{2}+\delta_{2}\right] I_{e}(t), \\
& \frac{d R}{d l t}=\gamma_{1} I_{u}(t)+\gamma_{2} I_{e}(t)-\mu R(t)
\end{aligned}
$$

The model (8) extends some SEIR models in the literature such as those in $[33,34,36]$ by

(i) replacing the mass action incidence function with Holling type II incidence function,

(ii) splitting the compartment of infected individuals into educated (counsel) and uneducated (noncounsel) infected individuals thereby allowing time-varying infection rate. The epidemiological implication of this assumption is that educated infected individuals transmit the disease at a reduced rate $(0<\eta<1)$ in comparison to the uneducated infected individuals (due to behavioral changes). For instance, In Zambia, the decline in HIV incidence since early 1990s is attributed to behavioral changes [37]. Public health education campaigns have also been successfully implemented in numerous countries and communities, such as Uganda, Thailand, Zambia, and the US gay community [38, 39]. Between 1991 and 1998, HIV prevalence dramatically declined in Uganda from 21 to $9.8 \%$ (with a corresponding reduction in nonregular sexual partners by $65 \%$ coupled with greater levels of awareness about HIV/AIDS [38]. The Ugandan programme fostered community mobilization towards change in risky behavior, without increasing stigma [40].

Further, unlike in the aforementioned modelling studies, detailed rigorous mathematical analysis of the model (8) will be provided.

2.1. Basic Properties. Since the model (8) monitors human populations, all its associated parameters are nonnegative. Further, the following nonnegativity result holds.

Theorem 1. The variables of the model (8) are nonnegative for all the time. In other words, solutions of the model system (8) with positive initial data will remain positive for all time $t>0$.

Proof. Let $t_{1}=\sup \left\{t>0: S>0, E>0, I_{u}>0, I_{e}>0, R>0 \in\right.$ $[0, t]\}$. Thus, $t_{1}>0$. It follows from the first equation of the system (8) that

$$
\frac{d S}{d t}=\Pi-\lambda(t) S(t)-\mu S(t) \geq \Pi-(\lambda+\mu) S(t),
$$

which can be rewritten as

$$
\frac{d}{d t}\left[S(t) \exp \left\{\mu t+\int_{0}^{t} \lambda(\tau) d \tau\right\}\right] \geq \Pi \exp \left\{\mu t+\int_{0}^{t} \lambda(\tau) d \tau\right\} .
$$

Hence,

$$
\begin{aligned}
& S\left(t_{1}\right) \exp \left\{\mu t_{1}+\int_{0}^{t} \lambda(\tau) d \tau\right\}-S(0) \\
& \geq \int_{0}^{t_{1}} \Pi \exp \left\{\mu y+\int_{0}^{y} \lambda(\tau) d \tau\right\} d y,
\end{aligned}
$$

so that

$$
\begin{aligned}
S(t) \geq & S(0) \exp \left\{-\mu t_{1}-\int_{0}^{t_{1}} \lambda(\tau) d \tau\right\} \\
& \times\left[\exp \left\{-\mu t_{1}-\int_{0}^{t_{1}} \lambda(\tau) d \tau\right\}\right] \\
& \times \int_{0}^{t_{1}} \Pi \exp \left\{\mu y+\int_{0}^{y} \lambda(\tau) d \tau\right\} d y>0 .
\end{aligned}
$$

Similarly, it can be shown that $E>0, I_{u}>0, I_{e}>0$ and $R>0$, for all time $t>0$. 
The previous result can also be established using the method in of [41, Appendix A].

We claim the following result.

Lemma 2. The closed set

$$
\mathscr{D}=\left\{\left(S, E, I_{u}, I_{e}, R\right) \in \mathbb{R}_{+}^{5}: S+E+I_{u}+I_{e}+R \leq \frac{\Pi}{\mu}\right\}
$$

is positively invariant.

Proof. Adding all the equations of the model (8) gives,

$$
\frac{d N}{d t}=\Pi-\mu N-\left(\delta_{1} I_{u}+\delta_{2} I_{e}\right)
$$

Since $d N / d t \leq \Pi-\mu N$, it follows that $d N / d t \leq 0$ if $N \geq \Pi / \mu$. Thus, a standard comparison theorem [42] can be used to show that $N \leq N(0) e^{-\mu t}+(\Pi / \mu)\left(1-e^{-\mu t}\right)$. In particular, $N(t) \leq \Pi / \mu$ if $N(0) \leq \Pi / \mu$. Thus, the region $\mathscr{D}$ is positively invariant. Further, if $N(0)>\Pi / \mu$, then either the solution enters $\mathscr{D}$ infinite time or $N(t)$ approaches $\Pi / \mu$ asymptotically. Hence, the region $\mathscr{D}$ attracts all solutions in $\mathbb{R}_{+}^{5}$.

Since the region $\mathscr{D}$ is positively invariant, it is sufficient to consider the dynamics of the flow generated by the model (8) in $\mathscr{D}$, where the usual existence, uniqueness, continuation results hold for the system [10].

\section{Local Stability of Disease-Free Equilibrium (DFE)}

The DFE of the model (8) is given by

$$
\varepsilon_{0}=\left(S^{*}, E^{*}, I_{u}^{*}, I_{e}^{*}, R^{*}\right)=\left(\frac{\Pi}{\mu}, 0,0,0,0\right) .
$$

The local stability of $\mathcal{E}_{0}$ will be explored using the next generation operator method $[43,44]$. Using the notation in [44], the nonnegative matrix, $F$, of the new infection terms and the $M$-matrix, $V$, of the transition terms associated with the model (8) are given, respectively, by

$$
\begin{gathered}
F=\left(\begin{array}{ccc}
0 & \frac{\beta \Pi}{\mu} & \frac{\eta \beta \Pi}{\mu} \\
0 & 0 & 0 \\
0 & 0 & 0
\end{array}\right), \\
V=\left(\begin{array}{ccc}
\mu+\kappa & 0 & 0 \\
-\kappa & \mu+\sigma+\gamma_{1}+\delta_{1} & 0 \\
0 & -\sigma & \mu+\gamma_{2}+\delta_{2}
\end{array}\right) .
\end{gathered}
$$

It follows that the control reproduction number [4, 21], denoted by $\mathcal{R}_{0}=\rho\left(F V^{-1}\right)$, where $\rho$ is the spectral radius, is given by

$$
\mathcal{R}_{0}=\frac{\beta \Pi \kappa\left(k_{3}+\eta \sigma\right)}{\mu k_{1} k_{2} k_{3}}
$$

where

$$
k_{1}=\mu+\kappa, \quad k_{2}=\mu+\sigma+\gamma_{1}+\delta_{1}, \quad k_{3}=\mu+\gamma_{2}+\delta_{2} .
$$

Using in [44, Theorem 2], the following result is established.

Lemma 3. The DFE of the model (8), given by (15), is locally asymptotically stable (LAS) if $\mathcal{R}_{0}<1$ and unstable if $\mathcal{R}_{0}>1$.

The quantity $\mathcal{R}_{0}$ measures the average number of new infections generated by a single infected individual in a population. Lemma 3 implies that the disease can be eliminated from the community (when $\mathcal{R}_{0}<1$ ) if the initial sizes of the subpopulations of the model are in the basin of attraction of the DFE $\left(\varepsilon_{0}\right)$. To ensure that disease elimination is independent of the initial sizes of sub-populations, it is necessary to show that the DFE is globally asymptotically stable (GAS) if $\mathcal{R}_{0}<1$. This is explored below.

\subsection{Global Stability of DFE}

Theorem 4. The DFE of the model (8), given by (15), is GAS in $\mathcal{D}$ whenever $\mathcal{R}_{0} \leq 1$.

Proof. Consider the following Lyapunov function:

$$
\mathcal{F}=\left(\frac{\kappa\left(k_{3}+\eta \sigma\right)}{\eta k_{1} k_{2}}\right) E+\left(\frac{k_{3}+\eta \sigma}{\eta k_{2}}\right) I_{u}+I_{e},
$$

with Lyapunov derivative (where a dot represents differentiation with respect to time) given by

$$
\begin{aligned}
\dot{\mathcal{F}}= & \left(\frac{\kappa\left(k_{3}+\eta \sigma\right)}{\eta k_{1} k_{2}}\right) \dot{E}+\left(\frac{k_{3}+\eta \sigma}{\eta k_{2}}\right) \dot{I}_{u}+\dot{I}_{e} \\
= & \left(\frac{\kappa\left(k_{3}+\eta \sigma\right)}{\eta k_{1} k_{2}}\right)\left[\beta S\left(\frac{I_{u}}{1+\alpha_{1} I_{u}}+\frac{\eta I_{e}}{1+\alpha_{2} I_{e}}\right)-k_{1} E\right] \\
& +\left(\frac{k_{3}+\eta \sigma}{\eta k_{2}}\right)\left[\kappa E-k_{2} I_{u}\right]+\sigma I_{u}-k_{3} I_{e} \\
\leq & \left(\frac{\kappa\left(k_{3}+\eta \sigma\right)}{\eta k_{1} k_{2}}\right)\left[\beta \frac{\Pi}{\mu}\left(I_{u}+\eta I_{e}\right)-k_{1} E\right] \\
& +\left(\frac{k_{3}+\eta \sigma}{\eta k_{2}}\right)\left[\kappa E-k_{2} I_{u}\right]+\sigma I_{u}-k_{3} I_{e} \\
= & \frac{\beta \Pi \kappa\left(k_{3}+\eta \sigma\right)}{\mu \eta k_{1} k_{2}}\left(I_{u}+\eta I_{e}\right)+\left(\sigma-\frac{k_{3}+\eta \sigma}{\eta}\right) I_{u}-k_{3} I_{e} \\
= & \frac{k_{3}}{\eta}\left(\mathcal{R}_{0}-1\right)\left(I_{u}+\eta I_{e}\right) .
\end{aligned}
$$

Since all the parameters and variables of the model (8) are nonnegative (Theorem 1), it follows that $\dot{\mathcal{F}} \leq 0$ for $\mathcal{R}_{0} \leq 1$ with $\dot{\mathcal{F}}=0$ if and only if $E=I_{u}=I_{e}=0$. Hence, $\mathcal{F}$ is a Lyapunov function on $\mathcal{D}$. Therefore, the largest compact invariant subset of the set where $\dot{\mathcal{F}}=0$ is the singleton 
$\left\{\left(E, I_{u}, I_{e}\right)=(0,0,0)\right\}$. Thus, it follows, by the LaSalle's invariance principle $[18,45]$, that

$$
\left(E, I_{u}, I_{e}\right) \longrightarrow(0,0,0) \quad \text { as } t \longrightarrow \infty .
$$

Since limsup $\sup _{t \rightarrow \infty} I_{u}=0$ and $\lim \sup _{t \rightarrow \infty} I_{e}=0$ (from (21)), it follows that, for sufficiently small $\varpi^{*}>0$, there exist constants $M_{1}>0$ and $M_{2}>0$ such that $\limsup _{t \rightarrow \infty} I_{u} \leq \omega^{*}$ for all $t>M_{1}$ and $\limsup _{t \rightarrow \infty} I_{e} \leq \omega^{*}$ for all $t>M_{2}$. Hence, it follows from the last equation of the model (8) that, for $t>\max \left\{M_{1}, M_{2}\right\}$,

$$
\dot{R} \leq \gamma_{1} \omega^{*}+\gamma_{2} \omega^{*}-\mu R .
$$

Thus, by comparison theorem [42],

$$
R^{\infty}=\limsup _{t \rightarrow \infty} R \leq \frac{\gamma_{1} \varpi^{*}+\gamma_{2} \varpi^{*}}{\mu},
$$

so that, by letting $\omega^{*} \rightarrow 0$,

$$
R^{\infty}=\limsup _{t \rightarrow \infty} R \leq 0 .
$$

Similarly (by using $\liminf _{t \rightarrow \infty} I_{u}=0$ and $\liminf _{t \rightarrow \infty} I_{e}=0$ ), it can be shown that

$$
R_{\infty}=\liminf _{t \rightarrow \infty} R \geq 0
$$

Thus, it follows from (24) and (25) that

$$
R_{\infty} \geq 0 \geq R^{\infty} .
$$

Hence,

$$
\lim _{t \rightarrow \infty} R=0 .
$$

Similarly, it can be shown that

$$
\lim _{t \rightarrow \infty} S(t)=\frac{\Pi}{\mu} .
$$

Thus, by combining (21), (27), and (28), it follows that every solution of the equations of the model (8), with initial conditions in $\mathscr{D}$, approaches $\varepsilon_{0}$ as $t \rightarrow \infty\left(\right.$ for $\left.\mathcal{R}_{0}<1\right)$.

The previous result shows that the disease can be eliminated from the community if the associated reproduction number of the model is less than unity.

\section{Existence and Stability for Endemic Equilibrium Point}

In this section, the possible existence and stability of endemic (positive) equilibria of the model (8) (i.e., equilibria where at least one of the infected components of the model is nonzero) will be explored.
4.1. Existence of Endemic Equilibrium Point (EEP). First of all, the persistence of the disease in the population will be investigated below. The model system (8) is said to be uniformly persistent if there exists a constant $c$ such that any solution $\left(S(t), E(t), I_{u}(t), I_{e}(t), R(t)\right)$ satisfies $([31,46])$

$$
\begin{gathered}
\liminf _{t \rightarrow \infty} S(t) \geq c, \quad \liminf _{t \rightarrow \infty} E(t) \geq c, \quad \liminf _{t \rightarrow \infty} I_{u}(t) \geq c, \\
\liminf _{t \rightarrow \infty} I_{e}(t) \geq c, \quad \liminf _{t \rightarrow \infty} R(t) \geq c,
\end{gathered}
$$

provided that $\left(S(0), E(0), I_{u}(0), I_{e}(0), R(0)\right) \in \mathscr{D}^{\circ}$ (the interior of the region $\mathscr{D})$.

Theorem 5. System (8) is uniformly persistent in $\mathscr{D}^{\circ}$ if and only if $\mathcal{R}_{0}>1$.

Proof. The theorem can be proved by using the approach used to prove Proposition 3.3 of [32], by applying a uniform persistence result in [46] and noting that the DFE of the model (8) is unstable whenever $\mathcal{R}_{0}>1$ (Lemma 3 ).

When $\mathcal{R}_{0}>1$, it follows (from Theorem (3)) that model (8) is uniformly persistent; by using in [47, Theorem 2.8.6] and in [42, Theorem D.3] it follows that the model (8) has at least one endemic equilibrium in $\mathscr{D}^{\circ}$. Thus the following result is established.

Lemma 6. The model (2) has at least one endemic equilibrium, given by $\varepsilon_{1}=\left(S^{* *}, E^{* *}, I_{u}^{* *}, I_{e}^{* *}, R^{* *}\right)$, whenever $\mathcal{R}_{0}>1$.

The uniqueness of the endemic equilibrium will be investigated at the end of the next subsection.

4.2. Global Stability of Endemic Equilibrium for Special Case. Here, the global asymptotic stability property of the endemic equilibrium of the model (8) is given for a special case when educated (counsel) infected individuals do not transmit infection $(\eta=0)$. The model (8), with $\eta=0$, then reduces to:

$$
\begin{aligned}
& \frac{d S}{d t}=\Pi-\left[\mu+\beta f\left(I_{u}\right)\right] S(t), \\
& \frac{d E}{d t}=\beta f\left(I_{u}\right) S(t)-[\mu+\kappa] E(t), \\
& \frac{d I_{u}}{d t}=\kappa E(t)-\left[\mu+\sigma+\gamma_{1}+\delta_{1}\right] I_{u}(t), \\
& \frac{d I_{e}}{d t}=\sigma I_{u}(t)-\left[\mu+\gamma_{2}+\delta_{2}\right] I_{e}(t) \\
& \frac{d R}{d t}=\gamma_{1} I_{u}(t)+\gamma_{2} I_{e}(t)-\mu R(t),
\end{aligned}
$$

where

$$
f\left(I_{u}\right)=\frac{I_{u}}{1+\alpha_{1} I_{u}} .
$$

The reproduction number of the model (30) is given by

$$
\mathcal{R}_{0 r}=\left.\mathcal{R}_{0}\right|_{\eta=0}=\frac{\beta \Pi \kappa}{\mu k_{1} k_{2}} .
$$

We claim the following result. 
Theorem 7. The endemic equilibrium of the reduced model, given by (30), is GAS in $\mathscr{D}^{\circ}$ if $\mathcal{R}_{0 r}>1$. Thus it is unique.

Proof. Consider the reduced model, given by (30). Let $\mathcal{R}_{0 r}>$ 1 , so that the associated endemic equilibrium exists. Further, consider the following nonlinear Lyapunov function:

$$
\begin{aligned}
\mathcal{F}= & -S^{* *}-S^{* *} \ln \left(\frac{S}{S^{* *}}\right)+E-E^{* *}-E^{* *} \ln \left(\frac{E}{E^{* *}}\right) \\
& +\frac{k_{1}}{\kappa}\left[I_{u}-I_{u}^{* *}-I_{u}^{* *} \ln \left(\frac{I_{u}}{I_{u}^{* *}}\right)\right]
\end{aligned}
$$

with Lyapunov derivative

$$
\begin{aligned}
\dot{\mathcal{F}}= & \dot{S}-\frac{S^{* *}}{S} \dot{S}+\dot{E}-\frac{E^{* *}}{E} \dot{E}+\frac{k_{1}}{\kappa}\left(\dot{I}_{u}-\frac{I_{u}^{* *}}{I_{u}} \dot{I}_{u}\right), \\
= & \Pi-\beta S f\left(I_{u}\right)-\mu S-\frac{S^{* *}}{S}\left(\Pi-\beta S f\left(I_{u}\right)-\mu S\right) \\
& +\beta S f\left(I_{u}\right)-k_{1} E-\frac{E^{* *}}{E}\left(\beta S f\left(I_{u}\right)-k_{1} E\right) \\
& +\frac{k_{1}}{\kappa}\left[\kappa E-k_{2} I_{u}-\frac{I_{u}^{* *}}{I_{u}}\left(\kappa E-k_{2} I_{u}\right)\right] \\
= & \Pi\left(1-\frac{S^{* *}}{S}\right)-\mu S\left(1-\frac{S^{* *}}{S}\right) \\
& +\beta S^{* *} f\left(I_{u}\right)-\frac{k_{1} k_{2}}{\kappa} I_{u}-\frac{E^{* *} \beta S f\left(I_{u}\right)}{E} \\
& +k_{1} E^{* *}-\frac{k_{1} I_{u}^{* *} E}{I_{u}}+\frac{k_{1} k_{2} I_{u}^{* *}}{\kappa} .
\end{aligned}
$$

It can be shown from (30) that, at endemic steady state,

$$
\begin{gathered}
\Pi=\left[\mu+\beta f\left(I_{u}^{* *}\right)\right] S^{* *}, \\
k_{1}=\frac{\beta f\left(I_{u}^{* *}\right) S^{* *}}{E^{* *}}, \\
k_{2}=\frac{\kappa E^{* *}}{I_{u}^{* *}} .
\end{gathered}
$$

Using the relations (35) in (34) gives

$$
\begin{aligned}
\dot{\mathcal{F}}= & \mu S^{* *}\left(2-\frac{S^{* *}}{S}-\frac{S}{S^{* *}}\right)-\frac{\left(S^{* *}\right)^{2} f\left(I_{u}^{* *}\right)}{S} \\
& +\beta S^{* *} f\left(I_{u}^{* *}\right)+\beta S^{* *} f\left(I_{u}\right)-\frac{\beta S^{* *} f\left(I_{u}^{* *}\right) I_{u}}{I_{u}^{* *}} \\
& -\frac{E^{* *} \beta S f\left(I_{u}\right)}{E}+\beta S^{* *} f\left(I_{u}^{* *}\right) \\
& -\frac{\beta S^{* *} I_{u}^{* *} f\left(I_{u}^{* *}\right) E}{I_{u} E^{* *}}+\beta S^{* *} f\left(I_{u}^{* *}\right) .
\end{aligned}
$$

Adding and subtracting $\beta S^{* *} f\left(I_{u}^{* *}\right)$ and $\beta S^{* *} f^{2}\left(I_{u}^{* *}\right) I_{u} /$ $f\left(I_{u}\right) I_{u}^{* *}$ in (36) gives

$$
\begin{aligned}
\dot{\mathcal{F}}= & \mu S^{* *}\left(2-\frac{S^{* *}}{S}-\frac{S}{S^{* *}}\right)+\beta S^{* *} f\left(I_{u}^{* *}\right) \\
& \times\left(4-\frac{S^{* *}}{S}-\frac{E^{* *} S f\left(I_{u}\right)}{E S^{* *} f\left(I_{u}^{* *}\right)}-\frac{I_{u}^{* *} E}{I_{u} E^{* *}}-\frac{f\left(I_{u}^{* *}\right) I_{u}}{f\left(I_{u}\right) I_{u}^{* *}}\right) \\
& +\beta S^{* *} f\left(I_{u}\right)-\frac{\beta S^{* *} f\left(I_{u}^{* *}\right) I_{u}}{I_{u}^{* *}}-\beta S^{* *} f\left(I_{u}^{* *}\right) \\
& +\frac{\beta S^{* *} f^{2}\left(I_{u}^{* *}\right) I_{u}}{f\left(I_{u}\right) I_{u}^{* *}} .
\end{aligned}
$$

Rewriting the last four terms of (37) in terms of $\Pi-\mu S^{* *}$ (by using the relations in (35)) gives

$$
\begin{aligned}
& \dot{\mathcal{F}}=\mu S^{* *}\left(2-\frac{S^{* *}}{S}-\frac{S}{S^{* *}}\right)+\beta S^{* *} f\left(I_{u}^{* *}\right) \\
& \times\left(4-\frac{S^{* *}}{S}-\frac{E^{* *} S f\left(I_{u}\right)}{E S^{* *} f\left(I_{u}^{* *}\right)}-\frac{I_{u}^{* *} E}{I_{u} E^{* *}}-\frac{f\left(I_{u}^{* *}\right) I_{u}}{f\left(I_{u}\right) I_{u}^{* *}}\right) \\
& +\frac{\left(\Pi-\mu S^{* *}\right) f\left(I_{u}\right)}{f\left(I_{u}^{* *}\right)}-\frac{\left(\Pi-\mu S^{* *}\right) I_{u}}{I_{u}^{* *}}-\left(\Pi-\mu S^{* *}\right) \\
& +\frac{\left(\Pi-\mu S^{* *}\right) f\left(I_{u}^{* *}\right) I_{u}}{f\left(I_{u}\right) I_{u}^{* *}} \\
& =\mu S^{* *}\left(2-\frac{S^{* *}}{S}-\frac{S}{S^{* *}}\right)+\beta S^{* *} f\left(I_{u}^{* *}\right) \\
& \times\left(4-\frac{S^{* *}}{S}-\frac{E^{* *} S f\left(I_{u}\right)}{E S^{* *} f\left(I_{u}^{* *}\right)}-\frac{I_{u}^{* *} E}{I_{u} E^{* *}}-\frac{f\left(I_{u}^{* *}\right) I_{u}}{f\left(I_{u}\right) I_{u}^{* *}}\right) \\
& +\left(\Pi-\mu S^{* *}\right)\left[\frac{f\left(I_{u}\right)}{f\left(I_{u}^{* *}\right)}-\frac{I_{u}}{I_{u}^{* *}}-1+\frac{f\left(I_{u}^{* *}\right) I_{u}}{f\left(I_{u}\right) I_{u}^{* *}}\right] \\
& =\mu S^{* *}\left(2-\frac{S^{* *}}{S}-\frac{S}{S^{* *}}\right)+\beta S^{* *} f\left(I_{u}^{* *}\right) \\
& \times\left(4-\frac{S^{* *}}{S}-\frac{E^{* *} S f\left(I_{u}\right)}{E S^{* *} f\left(I_{u}^{* *}\right)}-\frac{I_{u}^{* *} E}{I_{u} E^{* *}}-\frac{f\left(I_{u}^{* *}\right) I_{u}}{f\left(I_{u}\right) I_{u}^{* *}}\right) \\
& +\left(\Pi-\mu S^{* *}\right) \frac{I_{u}}{f\left(I_{u}\right)}\left[\frac{f\left(I_{u}\right)}{I_{u}}-\frac{f\left(I_{u}^{* *}\right)}{I_{u}^{* *}}\right] \\
& \times\left[\frac{f\left(I_{u}\right)}{f\left(I_{u}^{* *}\right)}-1\right] \text {. }
\end{aligned}
$$

Since $f\left(I_{u}\right)$ is an increasing function and $S^{* *} \leq \Pi / \mu$, it follows that

$$
\left(\Pi-\mu S^{* *}\right) \frac{I_{u}}{f\left(I_{u}\right)}\left[\frac{f\left(I_{u}\right)}{I_{u}}-\frac{f\left(I_{u}^{* *}\right)}{I_{u}^{* *}}\right]\left[\frac{f\left(I_{u}\right)}{f\left(I_{u}^{* *}\right)}-1\right] \leq 0,
$$


finally, since the arithmetic mean exceeds the geometric mean, then

$$
\begin{gathered}
\left(2-\frac{S^{* *}}{S}-\frac{S}{S^{* *}}\right) \leq 0, \\
\left(4-\frac{S^{* *}}{S}-\frac{E^{* *} S f\left(I_{u}\right)}{E S^{* *} f\left(I_{u}^{* *}\right)}-\frac{I_{u}^{* *} E}{I_{u} E^{* *}}-\frac{f\left(I_{u}^{* *}\right) I_{u}}{f\left(I_{u}\right) I_{u}^{* *}}\right) \leq 0 .
\end{gathered}
$$

Further, since all the model parameters are nonnegative, it follows that $\dot{\mathcal{F}} \leq 0$ for $\mathcal{R}_{0 r}>1$ with $\dot{\mathcal{F}}=0$ if and only if $S=S^{* *}, E=E^{* *}, I_{u}=I_{u}^{* *}$. Hence, $\mathcal{F}$ is a Lyapunov function on $\mathscr{D}^{\circ}$. Therefore, the largest compact invariant subset of the set where $\dot{\mathcal{F}}=0$ is the singleton $\left\{\left(S, E, I_{u}\right)=\left(S^{* *}, E^{* *}, I_{u}^{* *}\right)\right\}$. Thus, it follows, by the laSalle's invariance Principle $[18,45]$, that $S(t) \rightarrow S^{* *}, E(t) \rightarrow E^{* *}$ and $I_{u}(t) \rightarrow I_{u}^{* *}$ as $t \rightarrow \infty$. Since $\lim \sup _{t \rightarrow \infty} I_{u}=I_{u}^{* *}$, it follows that, for sufficiently small $\epsilon>0$, there exist constants $T_{1}>0$ such that $\limsup _{t \rightarrow \infty} I_{u} \leq I_{u}^{* *}+\epsilon$ for all $t>T_{1}$. It follows from the fourth equation of (30) that, for $t>T_{1}$,

$$
\dot{I}_{e}(t) \leq \sigma\left(I_{u}^{* *}+\epsilon\right)-k_{3} I_{e}
$$

Thus, by comparison theorem [42],

$$
I_{e}^{\infty}=\limsup _{t \rightarrow \infty} \leq \frac{\sigma\left(I_{u}^{* *}+\epsilon\right)}{k_{3}} .
$$

Hence, by letting $\epsilon \rightarrow 0$, we have

$$
I_{e}^{\infty}=\limsup _{t \rightarrow \infty} \leq \frac{\sigma I_{u}^{* *}}{k_{3}} .
$$

Similarly, by using $\liminf \operatorname{in}_{t \rightarrow \infty} I_{u}(t)=I_{u}^{* *}$, it can be shown that

$$
I_{e \infty}=\liminf _{t \rightarrow \infty} \geq \frac{\sigma I_{u}^{* *}}{k_{3}} .
$$

Thus, it follows from (44) and (45) that

$$
I_{e \infty} \geq \frac{\sigma I_{u}^{* *}}{k_{3}} \geq I_{e}^{\infty} .
$$

Hence, $\lim _{t \rightarrow \infty} I_{e}(t)=\sigma I_{u}^{* *} / k_{3}=I_{e}^{* *}$. In a similar way, it can be shown that $\lim _{t \rightarrow \infty} R(t)=R^{* *}$. Thus, every solution to the equations of the reduced model, with initial condition in $\mathscr{D}^{\circ}$, approaches the endemic equilibrium of the reduced system (30) as $t \rightarrow \infty$ for $\mathcal{R}_{0 r}>1$. This shows that the endemic equilibrium is unique.

\section{Conclusions}

In this paper, an SEIR epidemic model with a nonlinear (Holling type II) incidence rate is designed and analysed. Some of the theoretical and epidemiological findings of the study are as follows.

(i) The model (8) has a locally stable disease-free equilibrium whenever the associated reproduction number is less than unity. (ii) The DFE of the model (8) is shown to be globally asymptotically stable when $\mathcal{R}_{0}<1$.

(iii) The model (8) is uniformly persistent in $\mathscr{D}^{\circ}$ if and only if $\mathcal{R}_{0}>1$.

(iv) The endemic equilibrium of the reduced model (8), with $\eta=0$, is shown to be globally asymptotically stable, when it exists.

\section{Acknowledgments}

S. Garba acknowledges, with thanks, the support on part of the University of Pretoria Research and Development Project (RDP). The authors are grateful to the two anonymous reviewers and the handling editor for their constructive comments, which have enhanced the paper.

\section{References}

[1] R. M. Anderson and R. M. May, Eds., Infectious Diseases of Humans: Dynamics and Control, Oxford University Press, New York, NY, USA, 1991.

[2] E. Beretta and D. Breda, "An SEIR epidemic model with constant latency time and infectious period," Mathematical Biosciences and Engineering, vol. 8, no. 4, pp. 931-952, 2011.

[3] K. Dietz, "Transmission and control of arbovirus disease," in Epidemiology, K. L. Cooke, Ed., p. 104, SIAM, Philadelphia, Pa, USA, 1975.

[4] H. W. Hethcote, "Mathematics of infectious diseases," SIAM Review, vol. 42, no. 4, pp. 599-653, 2000.

[5] C. Rebelo, A. Margheri, and N. Bacaër, "Persistence in seasonally forced epidemiological models," Journal of Mathematical Biology, vol. 64, no. 6, pp. 933-949, 2012.

[6] Z. Feng and J. X. Velasco-Hernández, "Competitive exclusion in a vector-host model for the dengue fever," Journal of Mathematical Biology, vol. 35, no. 5, pp. 523-544, 1997.

[7] S. M. Garba and A. B. Gumel, "Mathematical recipe for HIV elimination in Nigeria," Journal of the Nigerian Mathematical Society, vol. 29, pp. 1-66, 2010.

[8] S. M. Garba, A. B. Gumel, and J. M. S. Lubuma, "Dynamicallyconsistent non-standard finite difference method for an epidemic model," Mathematical and Computer Modelling, vol. 53, no. 1-2, pp. 131-150, 2011.

[9] A. B. Gumel, "Global dynamics of a two-strain avian influenza model," International Journal of Computer Mathematics, vol. 86, no. 1, pp. 85-108, 2009.

[10] H. W. Hethcote and H. R. Thieme, "Stability of the endemic equilibrium in epidemic models with subpopulations," Mathematical Biosciences, vol. 75, no. 2, pp. 205-227, 1985.

[11] M. A. Safi and A. B. Gumel, "Mathematical analysis of a disease transmission model with quarantine, isolation and an imperfect vaccine," Computers and Mathematics with Applications, vol. 61, no. 10, pp. 3044-3070, 2011.

[12] M. A. Safi and A. B. Gumel, "Qualitative study of a quarantine/ isolation model with multiple disease stages," Applied Mathematics and Computation, vol. 218, no. 5, pp. 1941-1961, 2011.

[13] M. A. Safi, M. Imran, and A. B. Gumel, "Threshold dynamics of a non-autonomous SEIRS model with quarantine and isolation," Theory in Biosciences, vol. 131, no. 1, pp. 19-30, 2012.

[14] O. Sharomi, C. N. Podder, A. B. Gumel, E. H. Elbasha, and J. Watmough, "Role of incidence function in vaccine-induced 
backward bifurcation in some HIV models," Mathematical Biosciences, vol. 210, no. 2, pp. 436-463, 2007.

[15] T. Zhang and Z. Teng, "On a nonautonomous SEIRS model in epidemiology," Bulletin of Mathematical Biology, vol. 69, no. 8, pp. 2537-2559, 2007.

[16] L. D. Wang and J. Q. Li, "Global stability of an epidemic model with nonlinear incidence rate and differential infectivity," Applied Mathematics and Computation, vol. 161, no. 3, pp. 769-778, 2005.

[17] C. Vargas-De-León, "On the global stability of SIS, SIR and SIRS epidemic models with standard incidence," Chaos, Solitons \& Fractals, vol. 44, no. 12, pp. 1106-1110, 2011.

[18] J. K. Hale, Ordinary di Erential Equations, John Wiley \& Sons, New York, NY, USA, 1969.

[19] J. C. Kamgang and G. Sallet, "Computation of threshold conditions for epidemiological models and global stability of the disease-free equilibrium (DFE)," Mathematical Biosciences, vol. 213, no. 1, pp. 1-12, 2008.

[20] M. Qiao, A. Liu, and U. Foryś, "Qualitative analysis of the SICR epidemic model with impulsive vaccinations," Mathematical Methods in the Applied Sciences. In press.

[21] R. M. Anderson and R. M. Verlag, Population Biology of Infectious Diseases, Springer, New York, NY, USA, 1982.

[22] V. Capasso and G. Serio, "A generalization of the KermackMcKendrick deterministic epidemic model," Mathematical Biosciences, vol. 42, no. 1-2, pp. 43-61, 1978.

[23] J. Hou and Z. Teng, "Continuous and impulsive vaccination of SEIR epidemic models with saturation incidence rates," Mathematics and Computers in Simulation, vol. 79, no. 10, pp. 3038-3054, 2009.

[24] J. Zu, W. Wang, and B. Zu, "Evolutionary dynamics of prey-predator systems with holling type II," Mathematical Biosciences and Engineering, vol. 4, no. 2, pp. 221-237, 2007.

[25] W. M. Liu, S. A. Levin, and Y. Iwasa, "Influence of nonlinear incidence rates upon the behavior of SIRS epidemiological models," Journal of Mathematical Biology, vol. 23, no. 2, pp. 187-204, 1985.

[26] S. Ruan and W. Wang, "Dynamical behavior of an epidemic model with a nonlinear incidence rate," Journal of Differential Equations, vol. 188, no. 1, pp. 135-163, 2003.

[27] M. A. Safi and A. B. Gumel, "The effect of incidence functions on the dynamics of a quarantine/isolation model with time delay," Nonlinear Analysis: Real World Applications, vol. 12, no. 1, pp. 215-235, 2011.

[28] J. Zu, W. Wang, and B. Zu, "Evolutionary dynamics of preypredator systems with Holling type II functional response," Mathematical Biosciences and Engineering, vol. 4, no. 4, article $755,2007$.

[29] G. Huang and Y. Takeuchi, "Global analysis on delay epidemiological dynamic models with nonlinear incidence," Journal of Mathematical Biology, vol. 63, no. 1, pp. 125-139, 2011.

[30] H. R. Thieme, "Global asymptotic stability in epidemic models," in Equadiff, H. W. Knobloch and K. Schmidt, Eds., vol. 1017 of Lecture notes in Mathematics, pp. 608-615, Springer, Heidelberg, Germany, 1983.

[31] H. R. Thieme, "Epidemic and demographic interaction in the spread of potentially fatal diseases in growing populations," Mathematical Biosciences, vol. 111, no. 1, pp. 99-130, 1992.

[32] M. Y. Li, J. R. Graef, L. Wang, and J. Karsai, "Global dynamics of a SEIR model with varying total population size," Mathematical Biosciences, vol. 160, no. 2, pp. 191-213, 1999.

[33] M. Y. Li, H. L. Smith, and L. Wang, "Global dynamics of an seir epidemic model with vertical transmission," SIAM Journal on Applied Mathematics, vol. 62, no. 1, pp. 58-69, 2001.
[34] G. Li and Z. Jin, "Global stability of a SEIR epidemic model with infectious force in latent, infected and immune period," Chaos, Solitons and Fractals, vol. 25, no. 5, pp. 1177-1184, 2005.

[35] J. Zhang and Z. Ma, "Global dynamics of an SEIR epidemic model with saturating contact rate," Mathematical Biosciences, vol. 185, no. 1, pp. 15-32, 2003.

[36] A. Korobeinikov, "Global properties of SIR and SEIR epidemic models with multiple parallel infectious stages," Bulletin of Mathematical Biology, vol. 71, no. 1, pp. 75-83, 2009.

[37] K. Fylkesnes, R. M. Musonda, M. Sichone, Z. Ndhlovu, F. Tembo, and M. Monze, "Declining HIV prevalence and risk behaviours in Zambia: evidence from surveillance and population-based surveys," AIDS, vol. 15, no. 7, pp. 907-916, 2001.

[38] D. Low-Beer and R. L. Stoneburner, "Behaviour and communication change in reducing HIV: is Uganda unique?" African Journal of AIDS Research, vol. 2, no. 1, pp. 9-21, 2003.

[39] N. Hussaini, M. Winter, and A. B. Gumel, "Qualitative assessment of the role of public health education program on HIV transmission dynamics," Mathematical Medicine and Biology, vol. 28, no. 3, pp. 245-270, 2011.

[40] E. C. Green, D. T. Halperin, V. Nantulya, and J. A. Hogle, "Uganda's HIV prevention success: the role of sexual behavior change and the national response," AIDS and Behavior, vol. 10, no. 4, pp. 335-346, 2006.

[41] H. R. Thieme, Mathematics in Population, Princeton University Press, 2003.

[42] H. L. Smith and P. Waltman, The Theory of the Chemostat, Cambridge University Press, 1995.

[43] O. Diekmann, J. A. Heesterbeek, and J. A. Metz, "On the definition and the computation of the basic reproduction ratio R0 in models for infectious diseases in heterogeneous populations," Journal of Mathematical Biology, vol. 28, no. 4, pp. 365-382, 1990.

[44] P. Van Den Driessche and J. Watmough, "Reproduction numbers and sub-threshold endemic equilibria for compartmental models of disease transmission," Mathematical Biosciences, vol. 180, pp. 29-48, 2002.

[45] J. P. LaSalle, The Stability of Dynamical Systems, CBMS-NSF Regional Conference Series in Applied Mathematics, SIAM, Philadelphia, Pa, USA, 1976.

[46] H. I. Freedman, S. Ruan, and M. Tang, "Uniform persistence and flows near a closed positively invariant set," Journal of Dynamics and Differential Equations, vol. 6, no. 4, pp. 583600, 1994.

[47] N. P. Bhatia and G. P. Szeg, Dynamical Systems: Stability Theory and Applications, vol. 35 of Lecture Notes in Mathematics, Springer, Berlin, Germany, 1967. 


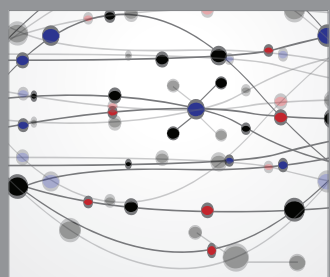

The Scientific World Journal
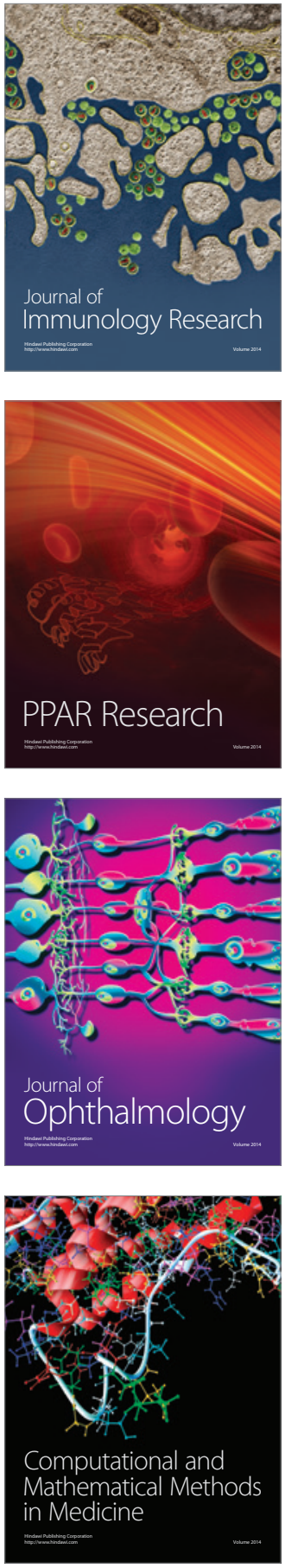

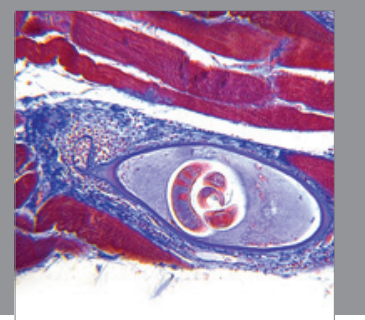

Gastroenterology

Research and Practice
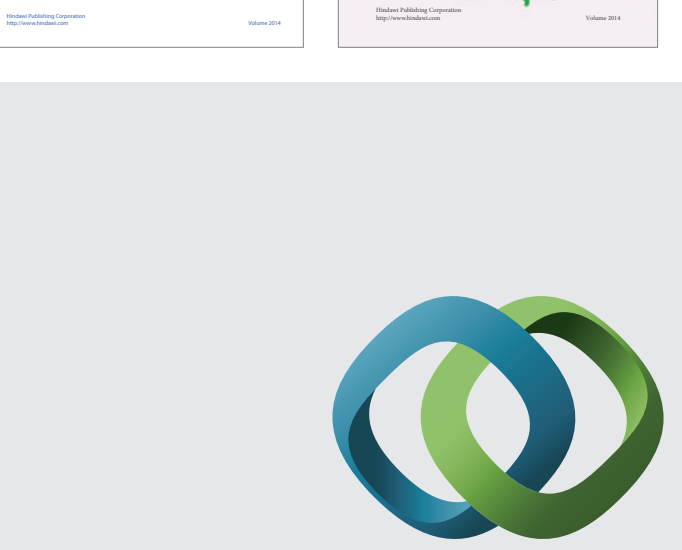

\section{Hindawi}

Submit your manuscripts at

http://www.hindawi.com
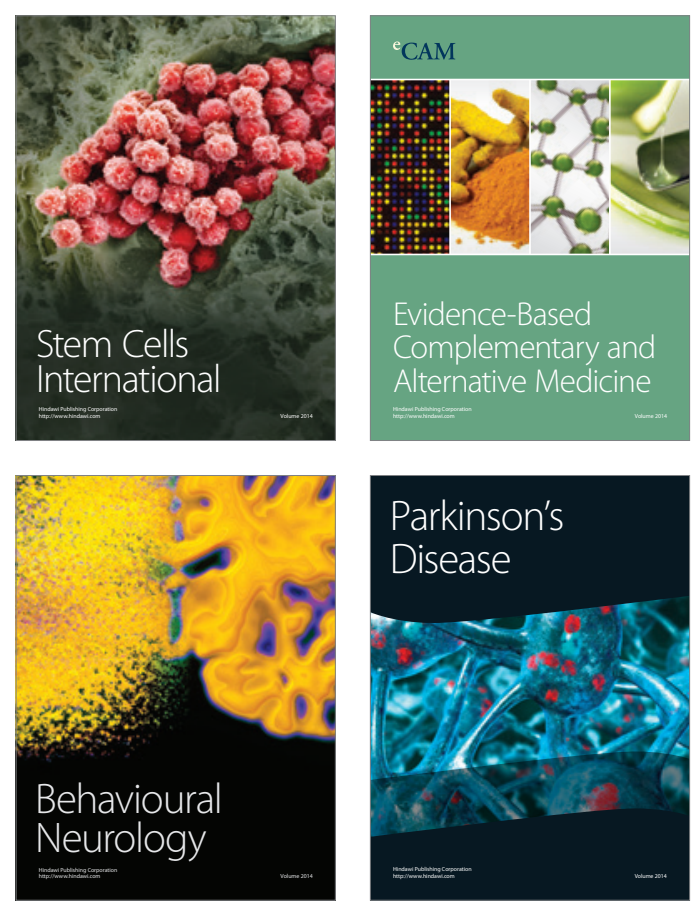

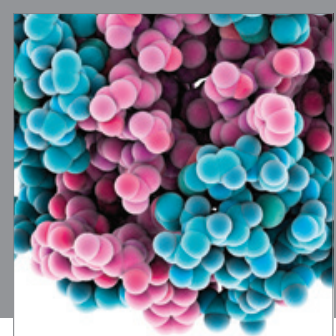

Journal of
Diabetes Research

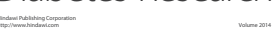

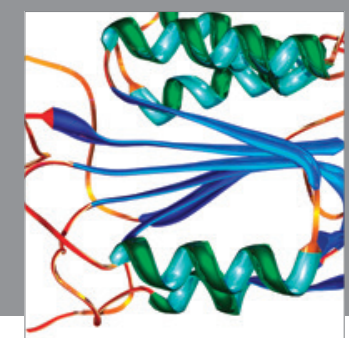

Disease Markers
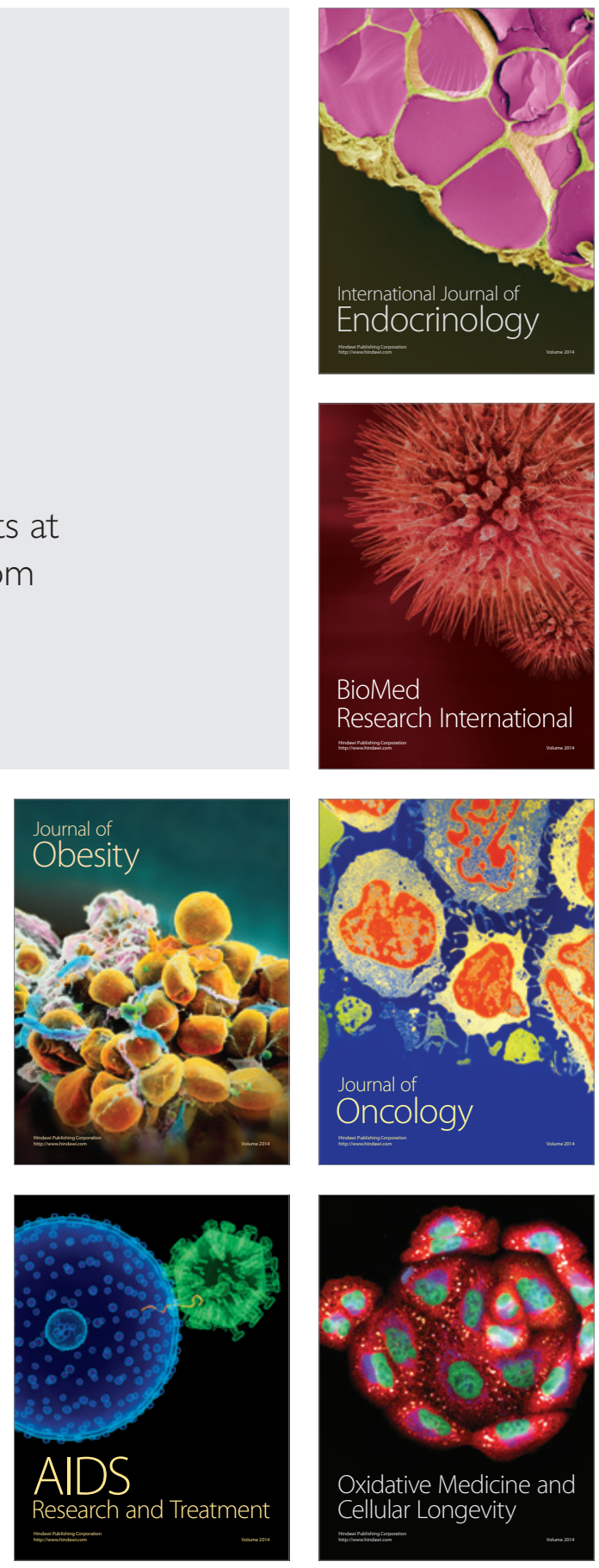